\title{
Pre-diagnostic drop out of presumptive TB patients and its associated factors at Bugembe Health Centre IV in Jinja, Uganda
}

\author{
Godfrey Ekuka ${ }^{1}$, Ismael Kawooya ${ }^{2,3}$, Edward Kayongo ${ }^{2,3}$, Ronald Ssenyonga ${ }^{4}$, Frank Mugabe ${ }^{5}$, \\ Peter Awongo Chaiga ${ }^{5}$, Andrew Nsawotebba ${ }^{1}$, Hannock Tweya ${ }^{6}$, Rhona Mijumbi-Deve ${ }^{2,3}$
}

\section{Emails:}

EG: goddiekuks@gmail.com; FM: rfmugabe@gmail.com; NA: andrewtebba@gmail.com;

SR:rssenyonga@musph.ac.ug; PA: peter.awongo@gmail.com; EK : kaydfirst@gmail.com;

IK:ikawooya@chs.mak.ac.ug; MRD:rmijumbi@chs.mak.ac.ug

1. National TB Reference Laboratory, Plot 106-1062 Butabika Road, Luzira, Kampala, Uganda

2. The Center for Rapid Evidence Synthesis (ACRES), Makerere University College of Health Sciences, Kampala, Uganda

3. Regional East African Community Health Policy Initiative (REACH-PI), Uganda Country node, Makerere University, Kampala Uganda

4. National TB and Leprosy Control Program, Ministry of Health, Plot 6, Lourdel Road, Nakasero, Kampala, Uganda

5. Clinical Trials Unit, Department of Epidemiology and Biostatistics, School of Public Health, Makerere University College of Health Sciences, Uganda

6. Light House Trust, Kamuzu Central Hospital Area 33 Mzimba Street, Malawi

\begin{abstract}
Background: Drop out of presumptive TB individuals before making a final diagnosis poses a danger to the individual and their community. We aimed to determine the proportion of these presumptive TB drop outs and their associated factors in Bugembe Health Centre, Jinja, Uganda.

Methods: We used data from the DHIS2, presumptive and laboratory registers of Bugembe Health Centre IV for 2017. Descriptive statistics were used to summarize the population characteristics. A modified Poisson regression model via the generalized linear model (GLM) with log link and robust standard errors was used for bivariate and multivariate analysis.

Results: Among the 216 registered presumptive TB patients who were less than $1 \%$ of patients visiting the outpatients' department, $40.7 \%$ dropped out before final diagnosis was made. Age and HIV status were significantly associated with pre-diagnostic drop out while gender and distance from the health center were not.

Conclusion: A high risk to individuals and the community is posed by the significant proportion of presumptive TB patients dropping out before final diagnosis. Health systems managers need to consider interventions targeting young persons, male patients, HIV positive persons.

Keywords: Tuberculosis (TB); Pre-diagnostic drop out; Presumptive TB; SORT IT.

DOI: https://doi.org/10.4314/ahs.v20i2.11

Cite as: Ekuka G, Kawooya I, Kayongo E, Ssenyonga R, Mugabe F, Chaiga PA, Nsawotebba A, Tweya H, Mijumbi-Deve R. Pre-diagnostic drop out of presumptive TB patients and its associated factors at Bugembe Health Centre IV in Jinja, Uganda. Afri Health Sci. 2020; 20(2): 633-640. https://doi.org/10.4314/abs.v20i2.11
\end{abstract}

\section{Corresponding author:}

Godfrey Ekuka,

Ministry of Health, Department of National

Disease Control

National TB Reference Laboratory

P.O. Box 16041 Wandegeya, Kampala Uganda

Telephone: +256-772-987699

Email: goddiekuks@gmail.com

\section{Introduction}

The Uganda National population Tuberculosis (TB) survey of 2014/2015 established that in addition to the TB prevalence and incidence in Uganda being higher than had previously been reported - 253/100,000 and $202 / 100,000$ respectively, much higher than previous estimates of 159/100,000 and 161/100,000 respectively - about half of the cases are missed each year ${ }^{1}$.

Amongst those cases that are missed, some are missed 
between the point at which they are considered presumptive and where they are fully diagnosed. An individual is considered presumptive at the health facility if they have one of the following: cough for 2 or more weeks; persistent fever for 2 or more weeks; noticeable weight loss; excessive night sweats and if it's a child, poor weight gain or history of contact with a PTB patient $^{2}$. In addition, they may be considered so if they are at high risk for TB (key population) and currently have a cough or - abnormal chest X-ray. Individuals lost between screening and confirmation of active TB are considered pre-diagnostic drop outs. Accurate diagnosis and successful treatment of TB averts millions of deaths annually - about 54 million lives were saved globally between 2000 and $2017^{3}$.

According to the 2014/15 National prevalence study, $39 \%$ of individuals with TB symptoms in Uganda did not seek care1. This included presumptive TB patients that might have dropped out of care during the diagnostic period, and diagnosed TB patients that might stop care before initiating treatment or while on a treatment. Elsewhere presumptive TB drop out has also been established as a major problem; in regions of Zimbabwe, India and Pakistan, the loss is estimated at 25\%,30\%, and $6-28 \%$ respectively ${ }^{4-6}$

Several factors have been associated with the loss between screening and final diagnosis, and they include patient-related factors such as poor health seeking behavior, lack of knowledge, poverty, long distances travelled, stigma, discrimination and low access to health services ${ }^{7,8}$. The national $\mathrm{TB}$ survey cited inadequate and overworked staff, long waiting times, disrespectful treatment, and drug stock-outs as contributors to poor quality care, deterring presumptive $\mathrm{TB}$ clients and known TB patients from seeking diagnosis and treatment at the health facility ${ }^{1}$.

Drop out of presumptive TB individuals before final diagnosis are made poses not only a danger to the individual, but is a public health risk as well. While the health of these individuals would deteriorate if they have active TB disease, they are also likely to transmit the disease in the community ${ }^{9-11}$.

This study aimed at assessing the pre-diagnostic drop out of presumptive TB cases and their associated factors at Bugembe HC IV, Jinja district. The study was commissioned by the TB supervising office of Bugembe after noticing several things in there host district: Jinja district has an incidence rate of 292/100,000, above the Uganda national average of 201/100,000, and clos- er to the upper limit of $302 / 100,000^{12}$. Furthermore, 1 in 3 of the expected TB cases in Jinja are not captured by the routine TB program ${ }^{13}$. The TB supervisory office of Bugembe therefore felt the need to explore several aspects on the TB situation in their sub-county, including the presumptive dropouts.

\section{Methods}

This was a cross sectional study among patients who were screened for $\mathrm{TB}$ at the outpatient department (OPD) at Bugembe Health center IV, Jinja District (Eastern Uganda) in 2017. The facility provides in- and out-patient care including TB diagnosis and treatment. The health facility is manned by about 37 medical personnel. The OPD received 40,032 people which is a half of its 83,279 catchment population ${ }^{14}$.

A presumptive $\mathrm{TB}$, and laboratory register are maintained at the facility. Patients with suggestive signs and symptoms are screened for TB and sent for a diagnostic test. The diagnosis of TB is confirmed bacteriologically using sputum smear microscopy, culture and/or Gene Xpert.

We included all patients who were recorded in the health facility's presumptive TB register and sent to the laboratory in 2017. We excluded those that appeared in the laboratory register but not the presumptive TB register as they may be referred cases not assessed by the Facility during that time.

Data collection: Data pertaining to the patients was extracted from the two registers. Individuals were considered to have dropped out if their names appeared in the presumptive TB register but not in the laboratory register. Biodata of each patient was also collected.

Data management and analysis: The data extracted was double-entered in EpiData and checked for completeness and accuracy before being exported to STATAV 14 for the analysis. Descriptive statistics were used to summarize the population characteristics. A modified Poisson regression model via the generalized linear model (GLM) with log link and robust standard errors was used for bivariate and multivariate analysis. Statistical significance was considered at a $\mathrm{p}$-value less thn 0.05 . We estimated to have a power of $86 \%$ at $\mathrm{p}<0.05$ and sample size of 216 assuming that the proportion of those expected to dropout before the laboratory were $30 \%$ and a difference of $10 \%$ in our setting.

Ethical approval for this study was obtained from Makerere University College of Health Sciences, School of Medicine Research and Ethics Committee (SOM-REC). 
Results

Forty thousand, thirty-two $(40,032)$ patients were received at the OPD in Bugembe HC IV between January and December 2017. Less than 1\% (216) were recorded in the presumptive TB register, of which $40.7 \%$ (88) did not reach the laboratory. (See figure 1)

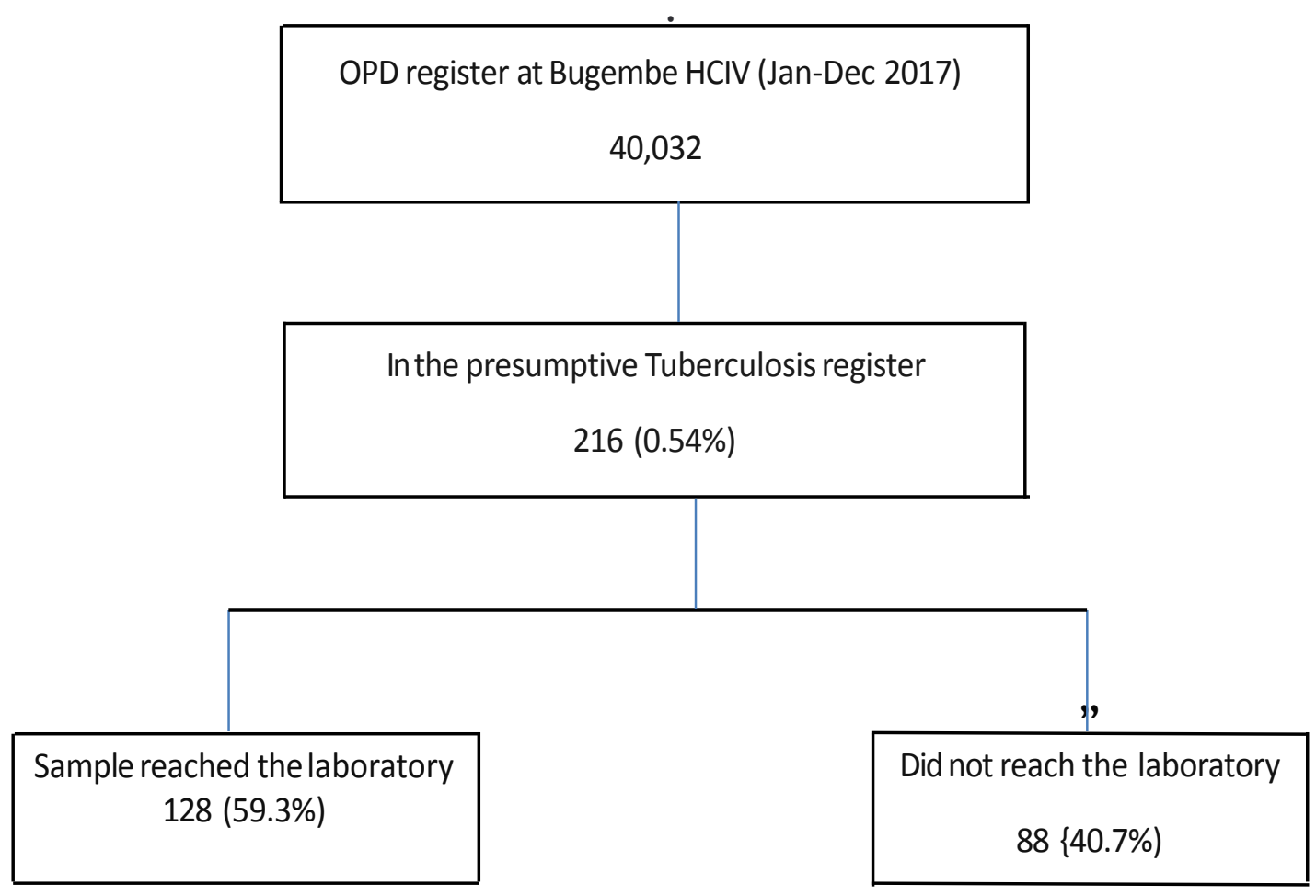

Table 1 presents the basic characteristics of the 216 between the ages of 18 and 31 years, $64.8 \%$ lived $1-4 \mathrm{~km}$ presumptive TB patients. The mean age of the patients from the health center, $54.2 \%$ were female and $39.4 \%$ was 31.5 [SD 13.0], majority (37.5\%) of whom were were HIV positive.

Table 1: Demographic characteristics of the study population

\begin{tabular}{lrc}
\hline Characteristics & \multicolumn{1}{c}{$\mathbf{N}(\%)$} \\
\hline Overall & $216(100)$ \\
Age: mean (SD) & $31.5(13.0)$ \\
Age in years & & \\
& Less than 18 & $33(15.3)$ \\
& $18-31$ & $81(37.5)$ \\
& $32-41$ & $47(21.8)$ \\
Sex & Greater than41 & $55(25.4)$ \\
& \\
& Female & $117(54.2)$ \\
Distance from Health facility (kilometers) & $99(45.8)$ \\
& $<1 \mathrm{~km}$ & $67(31.0)$ \\
& $1-5 \mathrm{~km}$ & $129(59.7)$ \\
HIV Sero status* & $>5 \mathrm{~km}$ & $20(9.3)$ \\
& \\
& Positive & $85(39.4)$ \\
& Negative & $131(60.6)$
\end{tabular}


Table 2 explores the factors associated with the dropping out of presumptive TB patients attending the OPD of Bugembe Health Center. Regarding age, the category less than 18 , category $32-41$ (OR 1.82, p value 0.014 ) and category above 41 (OR 1.88, P value 0.009) were significantly associated with pre-diagnostic drop out. Furthermore, HIV status (OR 1.43, p value -0.001 ) was significantly associated with pre-diagnostic drop out. Gender (OR 1.17. p-value 0.359) was not significantly associated. When it came to distance, in comparison to distance category less than $1 \mathrm{~km}$, category $1-5 \mathrm{~km}$ (OR 1.03, p value 0.808 ) and category above $5 \mathrm{~km}$ (OR 0.87 , $\mathrm{p}$ value 0.554 ) from the health center was not significantly associated with pre-diagnostic drop out.

Table 2: Bivariate and Multivariate analysis of the factors associated with presumptive TB patients drop out from diagnostic cascade at Bugembe Health Centre IV 2017

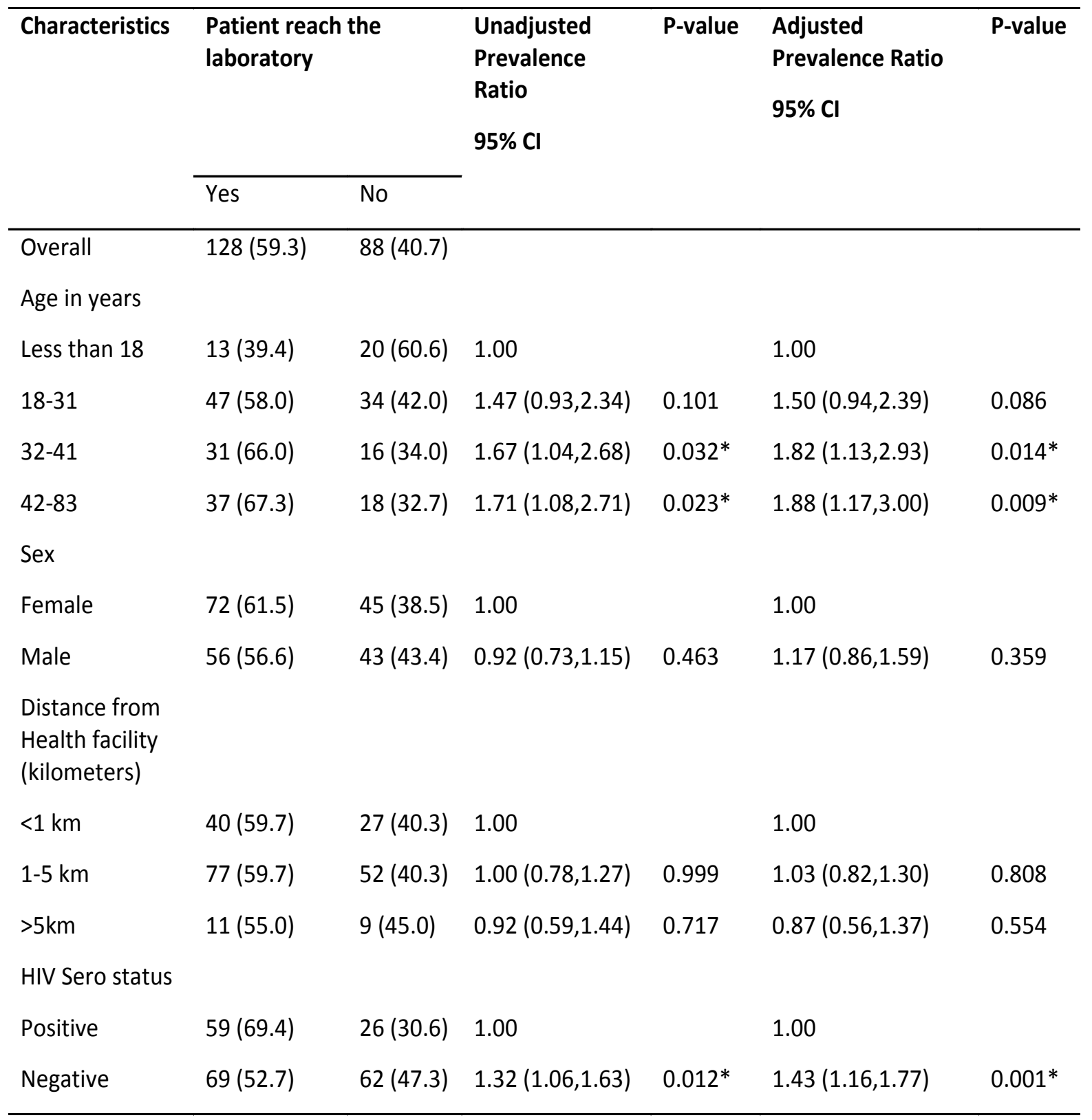

*Significant at $5 \%$ level of significance 


\section{Discussion}

This study aimed to determine the proportion and factors associated with dropout of registered presumptive TB patients before final diagnosis in Bugembe HC IV in 2017. We found that $40.7 \%$ of the presumptive TB patients did not reach the laboratory. Furthermore, age and HIV status were significantly associated with pre-diagnostic drop out while gender and distance from the health center were not.

\section{Interpretation of findings}

The revelation of a loss of 4 patients out of every 10 presumed to have TB is significant. This represents patients whose health is potentially at risk of deteriorating due to late diagnosis and delayed treatment. Worse still, a risk of transmitting the disease to the community which can have a ripple effect. Furthermore, this would affect the country economically by increasing the morbidity and in turn the cost of treatment and care $\cos ^{15}$, 16 .

\section{Situating in literature}

The proportion of presumptive TB drop outs we see in this study is significantly higher than that seen at the national level ${ }^{1}$. While the same proportion of $40 \%$ is reported for both presuptive TB and diagnosed patients who might stop care before initiating treatment or while on a treatment, in our study it referred to only presumptive TB patients. A study from Zimbabwe showed an overall pre-diagnostic loss to follow-up of $25 \% 4$ while another study from India indicated a $30 \%$ loss of presumptive TB patients before diagnosis was made ${ }^{5}$.

There were more female presumptive $\mathrm{TB}$ patients recorded, which is keeping with literature that shows that females with TB have better health seeking behavior than males ${ }^{17,18}$. In line with this, indeed the females in this study were less like to drop out of care before diagnosis, even if this was not statistically significant. This is similar to studies conducted elsewhere by Buregyeya and colleagues in Wakiso district, Uganda who found male gender as an independent predictor for patient delay ${ }^{19}$; and by Tesfahuneygn and colleagues who found male gender significantly associated with non-adherence to anti-TB regimens ${ }^{20}$.

We also found that the lower levels of age were more likely to drop out before diagnosis, especially those aged 18 years and below. The reasons for this may vary with a further break down of this age group. For example, children between 0-6 are difficult to diagnose and have their decisions managed by a care taker. Children in the adolescent age group may have to be in school or are involved in menial jobs that may not let them return to the health facility as expected. Pala and colleagues found that the presumptive TB dropout rate was higher among individuals below 15 years compared to those above 41 years in their study which intended to find out the proportion of presumptive pulmonary $\mathrm{TB}$ cases who were lost to follow up after consulting the physician for submission of two sputum specimens for diagnosis of pulmonary tuberculosis 5 , and so did Chanda-Kapata and colleagues in Zambia who found that older presumptive TB patients were more likely to seek care18. A study done by Barter and colleagues showed that TB patients may incur catastrophic costs in utilizing TB services and care and this can be a barrier for many young people seeking care and their families ${ }^{21}$. Ukwaja and colleagues note that these costs for TB care are potentially catastrophic for patients and households, even where services are provided free-of-charge ${ }^{22}$.

Those living $5 \mathrm{~km}$ and more from the health center were more likely to drop out before diagnosis. Indeed, the estimate in this study may even be an underestimate as GPS distances were considered as opposed to actual physical distances. The higher dropout rate coming with increasing distance may be explained by the difficulty that is involved in meeting costs of transport to the health facility. Socio-cultural differences may also begin to come into play as distances increase. A study done by Mauch and colleagues in Kenya noted that geographical and socio-cultural barriers do exist in accessing TB services ${ }^{23}$.

HIV negative individuals were more likely to make it to the point of diagnosis compared to their positive counterparts. Ansa and colleagues suggest that the HIV clients might drop out before diagnosis due to stigma(24). Furthermore anecdotal evidence shows that HIV positive patients need to attend several clinics for different reasons brought about by their status, especially in settings where these clinics have not been integrated, although the exact or full impact of integration continues to be investigated in low-income settings ${ }^{24}$.

\section{Implications for policy and practice}

With a prevalence of dropouts before TB diagnosis of 4 in every 10 patients, the risk to individuals and community is significantly high and calls for interventions to ensure that this is reduced. More targeted programs towards younger persons, male individuals and those living with HIV are important. Specifically, for individuals living with HIV, the call for more integrated services may contribute to the reduction of drop outs. There 
is also a need to think through what interventions may be appropriate for persons living far from the health facilities.

Other supportive interventions such as strengthening the counseling and preparation for presumptive TB patients, and improving the linkage to care to reduce loss to follow up before diagnosis, need to be considered.

\section{Implications for further research}

This study explored only the variables that are routinely collected by the health facilities which may not answer the research question fully. A study with a more appropriate design may be necessary to explore even more factors associated with presumptive TB dropout.

Furthermore, the study has highlighted the fact that the presumptive dropout rate in Bugembe is significantly higher than that at the national level. There is a need to explore the rates in different settings to ensure that the amount of resources directed towards a given problem actually tallies with the problem as contextualized for the given setting.

\section{Strengths and limitations}

This study is making use of the routinely collected data by the health center and regional hospital which is something that not many peripheral centers have made a habit of. It shows the benefit of using local evidence to contextualize problems and solutions.

In addition, this study is looking at one of the issues that is still of great concern - losing patients who are at great risk in the community. This may partly explain the slow reduction of incidences of the disease despite the different interventions in place.

Despite the strengths, this study was limited by the fact that we used routinely collected health facility data. This data may be incomplete and may have collected information about variables that may not completely answer the pertinent questions one has.

In this study we also did not review the laboratory registers of nearby health facilities where some presumptive TB patients could have been diagnosed. There is no known linked register which would highlight these cases and therefore one would need to explore them manually. This could mean an over-estimation of the presumptive TB dropout rate in this study.

As noted earlier, we calculated the patients' travel distance to the health center using GPS distance estimates which assume a straight line between the two points. This may underestimate distances if the actual route is not a straight one, which is morelikely in majority if not all of the distances measured which is likely to introduce a non-differential misclassification.

\section{Conclusion}

This study found a $40 \%$ prevalence of presumptive TB dropout rates in Bugembe HC IV, significantly higher than the national prevalence. In addition, it found that younger people (less than 18 years) and HIV positive people with presumptive TB were more likely to drop out before diagnosis. The study made use of the routinely collected health center data to highlight the problem and suggest interventions for target groups found to be prone to dropout, and also call for further research that would unearth more factors associated with this dropout. This would ensure better understanding of the problem and appropriately handling it so as to reduce on the risk of persons with transmittable $\mathrm{TB}$ in the community.

\section{Funding}

The program was funded by the United Kingdom's Department for International Development (DFID), The Union, MSF and La Fondation Veuve Emile MetzTesch (Luxembourg). La Fondation Veuve Emile MetzTesch supported open access publications costs. The funders had no role in study design, data collection and analysis, decision to publish, or preparation of the manuscript.

\section{Acknowledgement}

This research was conducted through the Structured Operational Research and Training Initiative (SORT IT), a global partnership led by the Special Programme for Research and Training in Tropical Diseases at the World Health Organization (WHO/TDR). The training model is based on a course developed jointly by the International Union Against Tuberculosis and Lung Disease (The Union) and Médecins sans Frontières (MSF). The specific SORT IT program which resulted in this publication was implemented by: The Ugandan country node of the Regional East African Community Health Policy Initiative (REACH-PI (U)) at Makerere University, Uganda.

We acknowledge the support of Mr. Luvaluka Richard, the TB focal person at Bugembe Health Center, Mr. Joseph Mukasa, the regional TB and Leprosy supervisor, Jinja Health region, Mr. Perez Kirya and Mrs. Rose Izizinga of the SORT IT team at Makerere University.

\section{Conflict of interest}

None declared.

\section{Open access statement}

In accordance with WHO's open-access publication policy for all work funded by WHO or authored / 
co-authored by WHO staff members, the WHO retains the copyright of this publication through a Creative Commons Attribution IGO license (http://creativecommons.org/licenses/by/3.0/igo/legalcode) which permits unrestricted use, distribution and reproduction in any medium provided the original work is properly cited.

\section{References}

1. Ministry of Health. The Uganda National Tuberculosis Prevalence Survey, 2014-2015 Survey Report In: National TB and Leprosy Programme, editor. Kampala, Uganda: $\mathrm{MoH} ; 2017$.

2. Ministry of Health. Algorithm for TB Screening, Diagnosis and Management. In: National Tuberculosis and Leprosy control Program, editor. Kampala, Uganda: $\mathrm{MoH} ; 2012$.

3. World Health Organisation. Global tuberculosis report 2018. Geneva: Licence: CC BY-NC-SA 3.0 IGO.; 2018.

4. Munekayi. Padingani, Ajay. Kumar, Jaya Prasad. Tripathy, Nyasha. Masuka, Sidingiliswe. Khumalo. Does pre-diagnostic loss to follow-up among presumptive TB patients differ by type of health facility? An operational research from Hwange, Zimbabwe in 2017. Pan African Medical Journal. 2018;31:196.

5. Pala S, Bhattacharya H, Lynrah KG, Sarkar A, Boro P, Medhi GK. Loss to follow up during diagnosis of presumptive pulmonary tuberculosis at a tertiary care hospital. Journal of Family Medicine and Primary Care. 2018;7(5):942-5.

6. Ali SM, Naureen F, Noor A, Fatima I, Viney K, Ishaq $\mathrm{M}$, et al. Loss-to-follow-up and delay to treatment initiation in Pakistan's national tuberculosis control programme. BMC Public Health. 2018;18(1):335.

7. Cattamanchi A, Miller CR, Tapley A, Haguma P, Ochom E, Ackerman S, et al. Health worker perspectives on barriers to delivery of routine tuberculosis diagnostic evaluation services in Uganda: a qualitative study to guide clinic-based interventions. BMC Health Services Research. 2015;15(1):10.

8. Ministry of Health. Revised National Strategic Plan 2015/16 - 2019/20. In: National Tuberculosis and Leprosy Control Program, editor. Kampala, Uganda June 2017.

9. Golub JE, Bur S Fau - Cronin WA, Cronin Wa Fau - Gange S, Gange S Fau - Baruch N, Baruch N Fau - Comstock GW, Comstock Gw Fau - Chaisson RE, et al. Delayed tuberculosis diagnosis and tuberculosis transmission. International Journal of Tuberculosis and Lung Disease. 2006;10(1):24-30.
10. Lonnroth K, Castro Kg Fau - Chakaya JM, Chakaya Jm Fau - Chauhan LS, Chauhan Ls Fau - Floyd K, Floyd K Fau - Glaziou P, Glaziou P Fau - Raviglione MC, et al. Tuberculosis control and elimination 201050: cure, care, and social development. The Lancet. 2010;375(9728):1814-29.

11. Uys PW, Warren Rm Fau - van Helden PD, van Helden PD. A threshold value for the time delay to TB diagnosis. PLOS One. 2007;2(8).

12. World Health Organisation. Tuberculosis Country Profile: Uganda2017. Available from: https://extranet. who.int $/$ sree $/$ Reports?op $=$ Replet\&name $=/ \mathrm{WHO}_{-}$ HQ_Reports/G2/PROD/EXT/TBCountryProfile\&ISO2 $=$ UG\&outtype $=$ PDF.

13. National TB and Leprosy Programme. League Table of District Performance. In: Ministry of Health, editor. Kampala, UgandaFY 2017/18.

14. Ministry of Health. Uganda eHMIS; Uganda's Electronic Helath Information System Kampala: Ministry of Health; 2018 [Available from: https://hmis2.health. go.ug/hmis2/dhis-web-commons/security/login.action? failed $=$ true.

15. Biya O, Gidado S, Abraham A, Waziri N, Nguku P, Nsubuga P, et al. Knowledge, care-seeking behavior, and factors associated with patient delay among newly-diagnosed pulmonary tuberculosis patients, Federal Capital Territory, Nigeria, 2010. The Pan African Medical Journal. 2014;18 Suppl 1(Suppl 1):6.

16. Mistry N, Rangan S, Dholakia Y, Lobo E, Shah S, Patil A. Durations and Delays in Care Seeking, Diagnosis and Treatment Initiation in Uncomplicated Pulmonary Tuberculosis Patients in Mumbai, India. PLoS One. 2016;11(3):e0152287.

17. Amo-Adjei J. Individual, household and community level factors associated with keeping tuberculosis status secret in Ghana. BMC Public Health. 2016;16(1):1196.

18. Chanda-Kapata P, Kapata N, Masiye F, Maboshe M, Klinkenberg E, Cobelens F, et al. Health Seeking Behaviour among Individuals with Presumptive Tuberculosis in Zambia. PLoS One. 2016;11(10):e0163975.

19. Buregyeya E, Criel B, Nuwaha F, Colebunders R. Delays in diagnosis and treatment of pulmonary tuberculosis in Wakiso and Mukono districts, Uganda. BMC Public Health. 2014;14(1):586.

20. Tesfahuneygn G, Medhin G, Legesse M. Adherence to Anti-tuberculosis treatment and treatment outcomes among tuberculosis patients in Alamata District, northeast Ethiopia. BMC Research Notes. 2015;8:503.

21. Barter DM, Agboola SO, Murray MB, Bärnighausen T. Tuberculosis and poverty: the contribution of pa- 
tient costs in sub-Saharan Africa--a systematic review. sis care with the Tool to Estimate Patients' Costs: pilot BMC Public Health. 2012;12:980.

results from two districts in Kenya. BMC Public Health.

22. Ukwaja KN, Alobu I, Lgwenyi C, Hopewell PC. 2011;11(1):43.

The high cost of free tuberculosis services: patient and 24. Ansa GA, Walley JD, Siddiqi K, Wei X. Assesshousehold costs associated with tuberculosis care in ing the impact of TB/HIV services integration on Ebonyi State, Nigeria. PLoS One. 2013;8(8):e73134. TB treatment outcomes and their relevance in TB/ 23. Mauch V, Woods N, Kirubi B, Kipruto H, Sitienei J, HIV monitoring in Ghana. Infectious Diseases of Poverty. Klinkenberg E. Assessing access barriers to tuberculo- 2012;1(1):13. 